\title{
Patulin Imprinted Nanoparticles Decorated Surface Plasmon Resonance Chips for Patulin Detection
}

\author{
Duygu ÇIMEN, Nilay BERELI, and Adil DENIZLI* \\ Department of Chemistry, Hacettepe University, Ankara 06800, Turkey \\ *Corresponding author: Adil DENIZLI ～E-mail: denizli@hacettepe.edu.tr
}

\begin{abstract}
In this study, the patulin imprinted and the non-imprinted nanoparticles are synthesized by the two-phase mini emulsion polymerization method and characterized by zeta-size analysis, Fourier transform infrared spectroscopy, and scanning electron microscopy. Afterwards, the patulin imprinted and the non-imprinted nanoparticles are attached on the surface of surface plasmon resonance (SPR) chips. The patulin imprinted and the non-imprinted SPR nanosensors are characterized by using atomic force microscope, ellipsometer, and contact angle measurements. Kinetic studies for patulin detection are carried out in the concentration range of $0.5 \mathrm{nmol}-750 \mathrm{nmol}$. The limit of detection and the limit of quantification values are obtained as $0.011 \mathrm{nmol}$ and $0.036 \mathrm{nmol}$, respectively. In all kinetic analysis, the response time is $13 \mathrm{~min}$ for equilibration, adsorption, and desorption cycles. The selectivity studies of the patulin imprinted and the non-imprinted SPR nanosensors are determined in the presence of ochratoxin A and aflatoxin B1. In order to demonstrate the applicability, validation studies of the patulin imprinted SPR nanosensor are performed by liquid chromatography-tandem mass spectrometry (LC-MS).
\end{abstract}

Keywords: Patulin; surface plasmon resonance; nanosensor; molecular imprinting technique

Citation: Duygu ÇIMEN, Nilay BERELI, and Adil DENIZLI, "Patulin Imprinted Nanoparticles Decorated Surface Plasmon Resonance Chips for Patulin Detection," Photonic Sensors, 2022, 12(2): 117-129.

\section{Introduction}

Mycotoxins are low molecular weight secondary metabolites produced by the fungal kingdom. Since most mycotoxins are resistant to digestion or breakdown, it is possible to encounter mycotoxins in meat or daily consumption products (such as eggs, cheese, and milk) [1,2]. Products contaminated by mycotoxins also have a negative impact on human health as they indirectly join the food chain. In addition, not only mycotoxins are limited to nutritional products, but also they can cause adverse effects on living things through ingestion, skin contact, and respiration [3, 4]. Mycotoxicity diseases are not contagious. However, antibiotics or drugs are not very effective. Spoilage of herbal products (nuts, spices, fruits, and fruit products) as a result of mycotoxin reproduction during harvest or pre-harvest, drying, and storage stages is a risk. Mycotoxins are produced by different fungal species (Aspergillus, Penicilium, Alternaria, Fusarium, and Claviceps) and because of this, they have a wide genetic diversity and each mycotoxin is responsible for various types of diseases [5-7].

Patulin (PAT) is a polar and hydrophilic molecule with a molecular weight of $154 \mathrm{~g} / \mathrm{mol}$. It is a secondary metabolite in the mycotoxin family known as toxic lactone [8]. Aspergillus, Penicillium,

Received: 22 March 2021 / Revised: 7 June 2021

(C) The Author(s) 2021. This article is published with open access at Springerlink.com DOI: $10.1007 / \mathrm{s} 13320-021-0638-1$

Article type: Regular 
Paecilomyces mushroom species and $P$. expansum, Bysochlamis nivea, and Aspergillus clavatus mushroom have determined the upper limit of the amount of patulin required in fruit or apple juices as $50 \mathrm{~g} / \mathrm{kg}$ and $10 \mathrm{~g} / \mathrm{kg}$ in baby food by the European Union [9-11]. Patulin has toxic side effects such as dermal, immunological, neurological, genotoxic, and gastrointestinal effects. Apart from its side effects, it has been reported that it may have mutagenic, carcinogenic, and teratogenic effects besides damaging organs such as kidneys, liver, brain, and immune system triggering oxidative DNA damage [12-14].

According to the Joint Food and Agriculture Organization/World Health Organization Expert Committee on Food Additives (JECFA), the provisional maximum tolerable dairly intake (PMTDI) amount for patulin is determined $0.4 \mu \mathrm{g} / \mathrm{kg}$ body weight/day. In European countries (EC), the patulin content of foods is determined very strictly. For a wide range of agricultural products, EC have introduced a limitation as follows: $50 \mu \mathrm{g} / \mathrm{kg}$ in fruit juices and other beverages, $25 \mu \mathrm{g} / \mathrm{kg}$ in solid products, and $10 \mu \mathrm{g} / \mathrm{kg}$ in apple products designed and labeled for infants and young children $[15,16]$.

Until now, various analytical methods such as tandem mass spectrometry-dependent liquid chromatography [17], capillary electrophoresis [18], liquid chromatography [19], and gas chromatographic mass spectrometry [20] have been used for patulin detection from fruit juice. While these methods have a strong analysis capability, high reproducibility, and sensitivity with low detection limits, they have many disadvantages such as time consuming, expensive instruments, and sample preparation steps that require trained personnel. The development of methods for detecting patulin is of great importance in food safety. Surface plasmon resonance (SPR) nanosensors have been used to detect patulin in recent years.

SPR nanosensors are based on measuring the change in refractive index of polarized light between two layers [21-24]. In the presence of a fixed wavelength and the thin metal layer on the surface, the SPR angle at which the resonance takes place depends on the refractive index of the material near the metal surface [25-28]. Label-free and simultaneous detection, fast analysis time, a very low amount sample and material consumption, high sensitivity and selectivity, and repetitive use of sensor chips are the most important advantages of SPR nanosensors. Besides, the disadvantages of SPR nanosensors are immobilization effects, sterile barrier with binding events, non-specific binding to surfaces, and cost of nanosensor chips and instrumentation [29-31]. SPR nanosensors are used in various fields for food quality control analysis, environmental analysis, and diagnostic purposes in medicine. As the validity of the SPR nanosensor technology in food analysis increases, the number of studies on target analyte determination in this field also increases. The targeted analytes in this area are pathogens, toxins, vitamins, hormones, allergens, proteins, and chemical contaminants [32-36].

In this study, the unique advantages of SPR nanosensors were combined with the molecular imprinting technique (MIT). Molecular imprinted polymers are highly advantageous among other receptors in which they are low-cost, easy to prepare, and highly selective and sensitive to the target molecule. The MIT was based on polymerization of the target molecule and functional monomers with a suitable initiator and crosslinker to form polymeric matrices [37]. When the target molecules were removed from polymeric matrices, the specific cavities were formed. When we looked at the studies performed in recent years, it has been seen that the MIT was applied from small molecules to larger biomolecules [38]. Stable synthetic polymers with selective molecular recognition regions were produced with a high stability, sensitivity, selectivity, reusablity, and low cost for the identification, determination, adsorption, and separation of 
molecules [39].

In this study, the patulin imprinted (MIP) and the non-imprinted (NIP) poly (hydroxyethyl methacrylate-methacrylic acid) [poly(HEMA-MAA)] nanoparticles were synthesized by the two-phase mini emulsion polymerization method and attached on the surface of the SPR chip. Also, the non-imprinted nanoparticles were prepared as a control without adding patulin and attached on the surface of the SPR chip. The patulin imprinted and the non-imprinted SPR nanosensors were characterized by ellipsometer, contact angle measurements, and atomic force microscope. The patulin imprinted SPR nanosensors were performed a real-time detection of patulin with kinetic analysis at different concentrations, selectivity, and reusability studies. Also, the applicability of the patulin imprinted SPR nanosensor in apple juice has been verified by comparison with liquid chromatography-tandem mass spectrometry (LC-MS).

\section{Experiment}

\subsection{Materials}

Patulin (PAT), ochratoxin A (OTA), aflatoxin B1 (AFB1), 2-hydroxyethyl methacrylate (HEMA), ethylene glycol dimethacrylate (EGDMA), methacrylic acid (MAA), sodium bisulfite (NaHSO3), poly vinyl alcohol (PVA), sodium bicarbonate $\left(\mathrm{NaHCO}_{3}\right)$, ammonium persulfate $\left[\left(\mathrm{NH}_{4}\right)_{2} \mathrm{~S}_{2} \mathrm{O}_{8}\right]$, and sodium dodecyl sulfate (SDS) were obtained from Sigma-Aldrich (St. Louis, Missouri, USA). SPR bare gold chips (SPRchip ${ }^{\mathrm{TM}}$, Masidon, WI, USA) were supplied for the SPRimager II instrument by GWC Technologies (Masidon, WI, USA).

\subsection{Preparation and characterization of the patulin imprinted and the non-imprinted nanoparticles}

The MIP and the NIP poly (hydroxyethyl methacrylate-methacrylic acid) [poly(HEMA-MAA)] nanoparticles were synthesized by using the two-phase mini emulsion polymerization method [40]. The first aqueous phase was prepared by dissolving $0.02 \mathrm{~g}$ of polyvinyl alcohol (PVA) as the stabilizer and $0.05 \mathrm{~g}$ of sodium dodecyl sulfate (SDS) as the surfactant in $25 \mathrm{~mL}$ of deionized water. The second phase, $93.5 \mathrm{mg}$ PVA, $14 \mathrm{mg}$ SDS, and $12.5 \mathrm{mg}$ sodium bicarbonate $\left(\mathrm{NaHCO}_{3}\right)$, was dissolved in $5 \mathrm{~mL}$ water. The pre-polymerization complex consisting of 10:1 mmol for MAA:PAT was prepared with methacrylic acid (MAA) (2 mmol) as the monomer and patulin $(0.2 \mathrm{mmol})$ as the template molecule for $2 \mathrm{~h}$. $0.5 \mathrm{~mL}$ of 2-hydroxyethyl methacrylate (HEMA) and $1.0 \mathrm{~mL}$ of ethylene glycol dimethacrylate (EGDMA), were added to the prepared pre-polymerization complex (MAA:PAT) and mixed for $1 \mathrm{~h}$. Afterwards, the organic phase was slowly added to the first aqueous phase and homogenized with each other at $6000 \mathrm{rpm}$ for $30 \mathrm{~min}$. Then, the mixture was added to the second aqueous phase while the final phase had been stirring in a sealed-cylindrical reactor. Finally, $50 \mathrm{mg}$ of sodium bisulfite $\left(\mathrm{NaHSO}_{3}\right)$ and $100 \mathrm{mg}$ of ammonium persulfate were added to this mixture as the initiator pair. The polymerization was carried out at $500 \mathrm{rpm}$ for $24 \mathrm{~h}$ at a temperature of $40{ }^{\circ} \mathrm{C}$. The non-imprinted nanoparticles were synthesized under the same experimental conditions without adding the template molecule patulin. The MIP and the NIP nanoparticles were removed from unreacted monomers by washing the deionized water and water/ethanol mixture.

The MIP and the NIP poly (hydroxyethyl methacrylate-methacrylic acid) [poly(HEMA-MAA)] nanoparticles were characterized by Fourier transform infrared (FTIR) spectroscopy (Thermo Fisher Scientific, Nicolet iS10, Waltham, MA, USA) for the chemical structure and zeta-sizer (Nano-ZS, Malvern Instrument Company, UK) for size distribution and electron microscope scanning (SEM, Quanta 400F Field Emission, USA). 


\subsection{Preparation and characterization of the patulin imprinted and the non-imprinted SPR nanosensors}

The gold surface of the SPR chip was washed with an ethanol/water mixture and dried. Then, $5 \mu \mathrm{L}$ of allyl mercaptan solution was dropped on the surface of the SPR chip. The gold surface of the SPR chip was washed with an ethanol/water mixture and dried at room temperature. $5 \mu \mathrm{L}$ of the patulin imprinted nanoparticle solution was dropped on the surface of the SPR chip and dispersed with a spin coater (LAURELL, WS 650Mz-23NPP, USA). The attachment of the patulin imprinted and the nonimprinted nanoparticles on the surface of the SPR chip was carried out under ultraviolet light $(365 \mathrm{~nm}$, $100 \mathrm{~W}$ ) for $20 \mathrm{~min}$ and incubated in an oven overnight at $40{ }^{\circ} \mathrm{C}$ to stabilize attachment. The preparation of the patulin imprinted SPR chip was shown in Fig. 1.

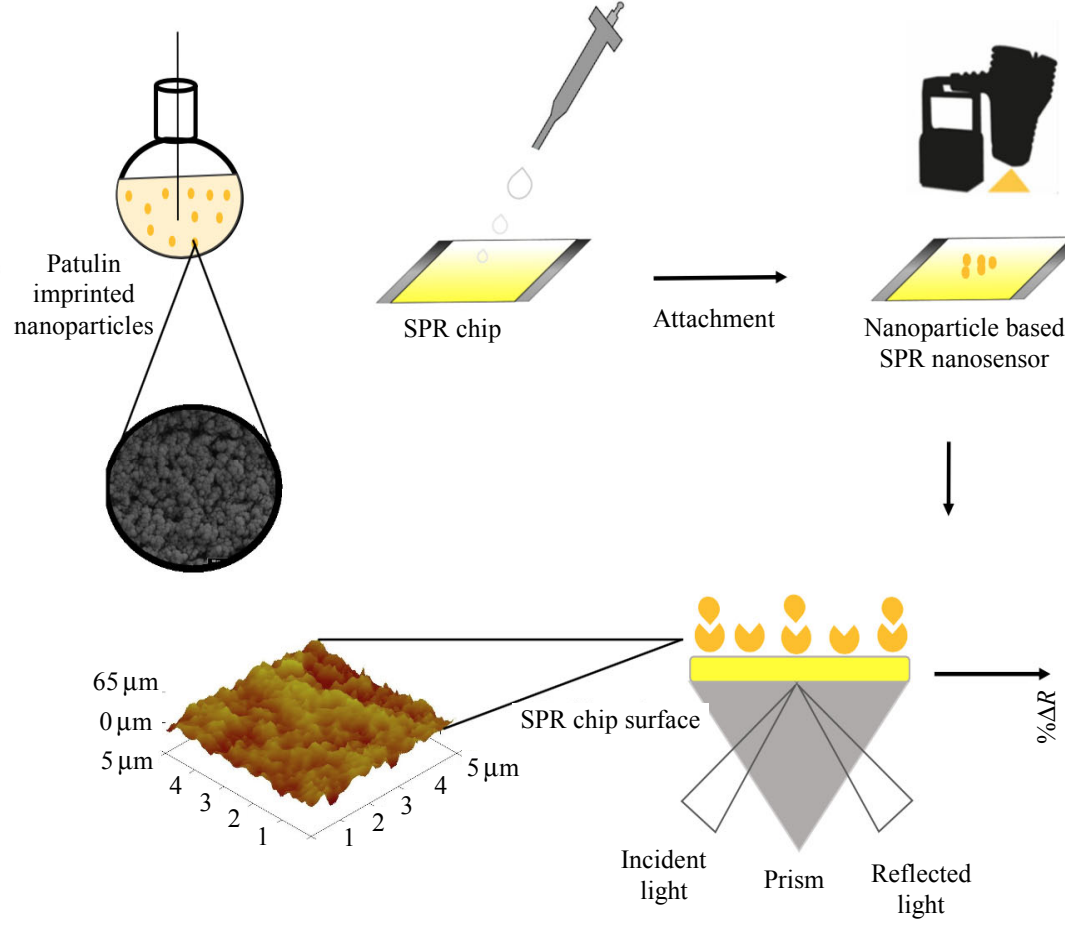

Fig. 1 Schematic illustration of the preparation of the patulin imprinted SPR nanosensor.

The characterization studies of the patulin imprinted and the non-imprinted SPR nanosensor surfaces were examined by the ellipsometer, contact angle measurements, and atomic force microscopy. For the hydrophilic characterization of the patulin imprinted and the non-imprinted SPR nanosensor surfaces, the contact angles were characterized by using the KRUSS device (Hamburg, Germany). The hydrophilic characterizations of the SPR nanosensor surfaces were obtained by dropping water to different parts of the SPR chip surface by using the sessile drop method. The morphology of the SPR chip surface was examined by using an atomic force microscope (Nanomagnetics Instruments, Oxford, UK) in a tapping mod with $1 \mu \mathrm{m} \times 1 \mu \mathrm{m}$ and $5 \mu \mathrm{m} \times$ $5 \mu \mathrm{m}$ area samples at $1 \mu \mathrm{m} / \mathrm{s}$ scanning speed and $256 \times 256$ pixel resolution. An automatic nulling imaging ellipsometer (Nanofilm EP3, Germany) at $62^{\circ}$ incidence and wavelength of $532 \mathrm{~nm}$ was used to examine the thicknesses of the SPR chip surfaces.

\subsection{Kinetic analysis}

Kinetic analyses were performed by using SPRimager II to detect patulin from both the aqueous solution and apple juice. For kinetic analysis, different patulin concentrations between $0.5 \mathrm{nmol}$ and $750 \mathrm{nmol}$ were prepared in $\mathrm{pH} 6.0$ 
phosphate buffer. Firstly, the patulin imprinted SPR nanosensor was equilibrated for $3 \mathrm{~min}$ with equilibration buffer ( $\mathrm{pH}$ 6.0, phosphate buffer). The prepared patulin solutions in different concentrations were applied to the SPR nanosensor for $7 \mathrm{~min}$. After each analysis, the desorption step was carried out using $0.5 \mathrm{~mol} \mathrm{NaCI}$ solution for $3 \mathrm{~min}$. In all kinetic analysis, equilibration-adsorption-desorption steps were carried out at $13 \mathrm{~min}$ and monitored the percent change in reflectivity values $(\% \Delta R)$ of the patulin imprinted SPR nanosensor.

OTA and AFB1 molecules were used as competitive agents to determine the specificity and selectivity of the patulin imprinted and the non-imprinted SPR nanosensors. Both molecular weights and structures of both ochratoxin A (MW: $403.813 \mathrm{~g} / \mathrm{mol}$ ) and aflatoxin B1 (MW: $312.27 \mathrm{~g} / \mathrm{mol}$ ) molecules were close to patulin (MW: $534.36 \mathrm{~g} / \mathrm{mol}$ ). Patulin, ochratoxin A, and aflatoxin B1 solutions were prepared with the same concentration $(100 \mathrm{nmol})$ and their interactions with the patulin imprinted and the non-imprinted SPR nanosensors were examined separately to compare the selectivity behavior. Selectivity $(k)$ and relative selectivity $\left(k^{\prime}\right)$ coefficients were calculated according to kinetic analysis of the patulin imprinted and the non-imprinted SPR nanosensors. $k$ and $k^{\prime}$ are described by the following equations [34]:

$$
\begin{gathered}
k=\Delta R_{\text {template }} / \Delta R_{\text {competitor }} \\
k^{\prime}=k_{(\mathrm{MIP})} / k_{(\mathrm{NIP})}
\end{gathered}
$$

where $\Delta R_{\text {template }}$ is the refractive index change of template molecule (patulin), and $\Delta R_{\text {competitor }}$ is the refractive index change of competitive agents (ochratoxin A and aflatoxin B1). Patulin solution with the same concentration was given to the SPR system five times to test the reusability performance of the patulin imprinted SPR nanosensor to determine the multiple usage and long shelf life.

\subsection{Patulin extraction from apple juice}

The applicability studies of the patulin imprinted SPR nanosensor were performed with apple juice samples. Apple juice was used as the real sample to determine patulin and obtained from the local market. $2 \mathrm{~mL}$ of apple juice was added to $5 \mathrm{~mL}$ centrifuge tube. It was extracted twice with $3 \mathrm{~mL}$ of ethyl acetate for $5 \mathrm{~min}$ to remove proteins, vitamins, polyphenols, carbohydrates, and organic acids from the apple juice sample. After this treatment, the ethyl acetate layers of each sample were removed and the liquid evaporated under a stream of $\mathrm{N}_{2}$. Then, $1.0 \mathrm{~mL}$ of $0.1 \mathrm{~mol}$ (pH 6.0) phosphate buffer was added to dissolve the dried residue. $5 \mathrm{~mL}$ of the remaining solution from the extracts was passed through solid phase extraction cartridges. $50 \mathrm{nmol}$ and $100 \mathrm{nmol}$ patulin standard solutions were spiked to the obtained extraction and the amount of patulin in the apple juice was determined by sensorgrams [41]. Also, the spiked apple juice samples were analyzed with liquid chromatography-tandem mass spectrometry (LC-MS) (Thermo Scientific TSQ Quantum Access Triple Quadrupole Cihaz, San Jose, CA, USA). Waters XBridge C18 column $(2.1 \mathrm{~mm} \times$ $50 \mathrm{~mm}, 1.8 \mu \mathrm{m})$ was used for detection of patulin. The mobile phase was deionized water $(\mathrm{A})$ and $\mathrm{ACN}$ (B). The flow rate was $0.25 \mathrm{~mL} \cdot \mathrm{min}^{-1}$ and at $25^{\circ} \mathrm{C}$, and the injection volume was $5 \mu \mathrm{L}$ [42].

\section{Results and discussion}

\subsection{Characterization studies}

The characterization studies of the patulin imprinted and the non-imprinted nanoparticles were made with zeta-sizer, SEM, and FTIR spectroscopy. The average nanoparticle size and polydispersity index (PDI) were measured for patulin imprinted nanoparticles by using the zeta-sizer. The average nanoparticle size was measured as $60.08 \mathrm{~nm}$ with 0.120 polydispersity index [Fig. 2(a)]. SEM analysis of the patulin imprinted nanoparticles was observed to have a rough surface with a size of about $60 \mathrm{~nm}$ [Fig. 2(b)]. The results of the SEM and zeta-sizer show that the molecular imprinted technique is successful in nanoparticle synthesis. The chemical 
structure of the MIP and the NIP poly (hydroxyethyl methacrylate-methacrylic acid [poly(HEMA-MAA)] nanoparticles was used by the FTIR spectroscopy.

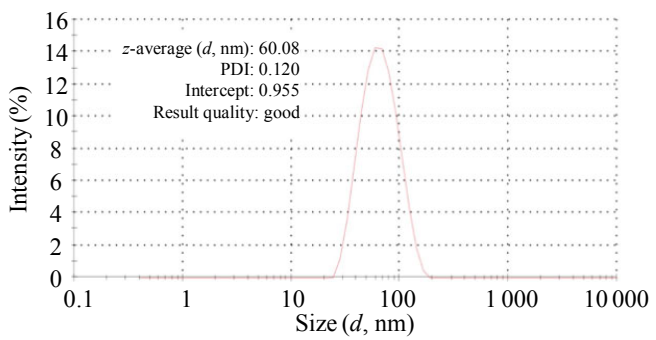

(a)

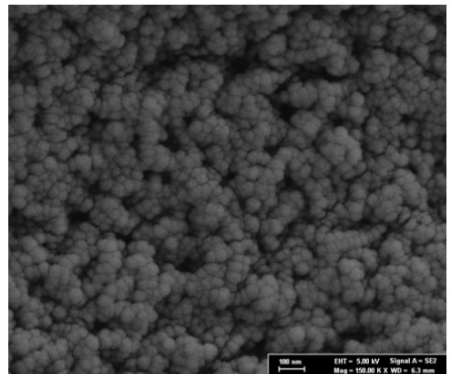

(b)

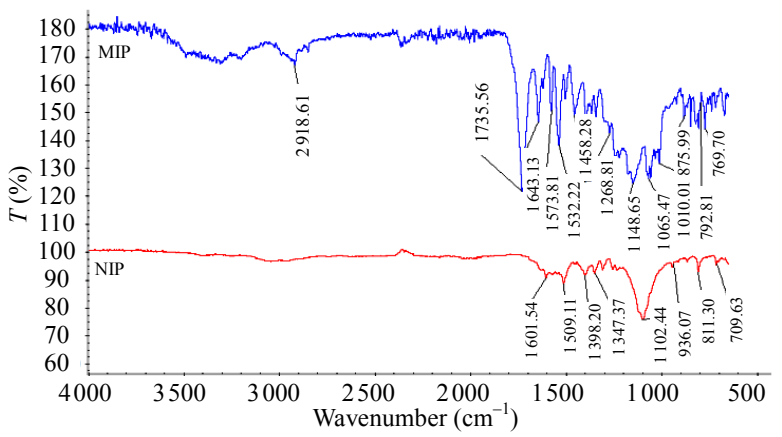

(c)

Fig. 2 Characterization analyses of the patulin imprinted nanoparticles: (a) zeta size measurement, (b) SEM, and (c) FTIR.

FTIR spectra of the patulin imprinted and the non-imprinted nanoparticles were determined in the frequency region $500 \mathrm{~cm}^{-1}-4000 \mathrm{~cm}^{-1}$ in Fig. 2(c). FTIR spectra of the patulin imprinted nanopaticles show several peaks at $2950 \mathrm{~cm}^{-1}$ (aliphatic $\mathrm{C}-\mathrm{H}$ stretching band), at $1730 \mathrm{~cm}^{-1}$ (carbonyl band), at $1573 \mathrm{~cm}^{-1}$ (COO- groups), $1458 \mathrm{~cm}^{-1}$ (C-N stretch), and $1065 \mathrm{~cm}^{-1}$ (strong aromatic $\mathrm{C}-\mathrm{H}$ stretching). These results showed that the patulin imprinted nanoparticles successfully got loaded the patulin polymer structure compared with non-imprinted nanoparticles [Fig.2(c)].

In addition, atomic force microscope (AFM) was used for the surface morphology of the bare gold
SPR chip, the patulin imprinted, and the non-imprinted SPR nanosensors in tapping mode. The surface depth values of the bare gold SPR chip, the patulin imprinted, and the non-imprinted SPR nanosensor surfaces were $3.94 \mathrm{~nm}, 65 \mathrm{~nm}$, and $61 \mathrm{~nm}$, respectively [Figs.3(a), 3(b), and 3(c)]. As can be seen from the AFM results, the patulin imprinted nanoparticles were spread homogeneously on the surface of the SPR nanosensor.

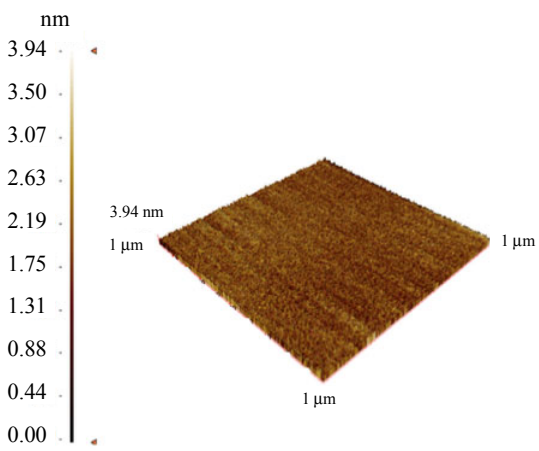

(a)

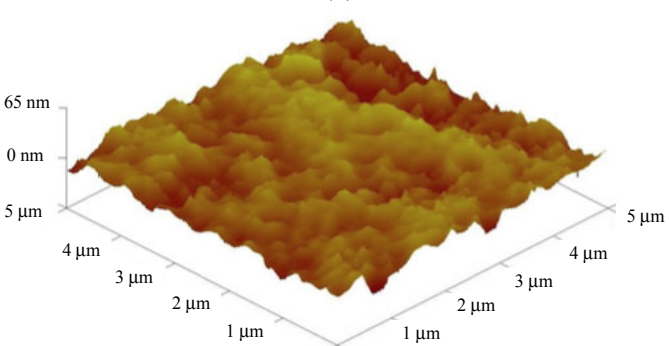

(b)

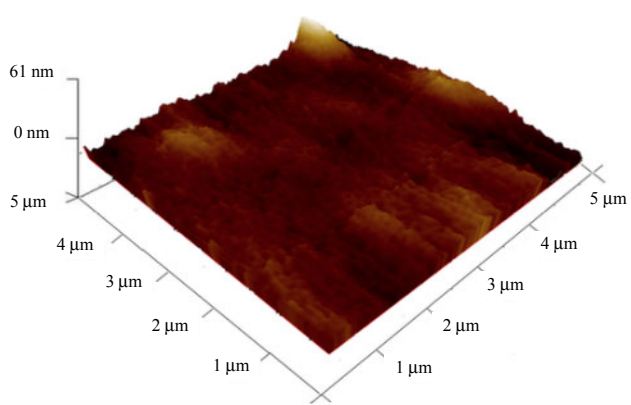

(c)

Fig. 3 AFM images of (a) bare gold SPR chip surface, (b) patulin imprinted SPR nanosensor surface, and (c) non-imprinted SPR nanosensor surface.

The contact angle values of the bare gold SPR chip surface, the patulin imprinted, and the non-imprinted SPR nanosensor surfaces were obtained as $80.2^{\circ}, 75.2^{\circ}$, and $70.3^{\circ}$, respectively [Figs. 4(a), 4(b), and 4(c)]. MAA monomer with hydrophilic structure was used in the patulin 
imprinted and the non-imprinted nanoparticle synthesis. The observed decrease in contact angle values depended on an increase in the hydrophilicity of the SPR surfaces. An increase in the hydrophilic character of the SPR surface was expected due to the hydrophilic functional groups of MAA monomer.

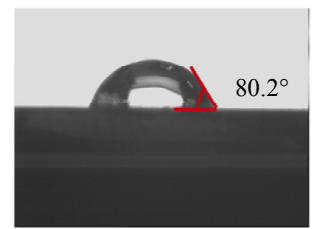

(a)

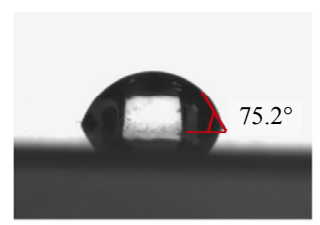

(b)

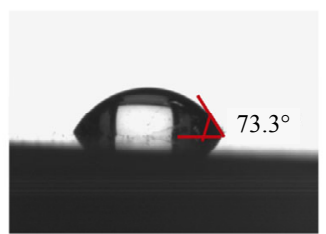

(c)

Fig. 4 Contact angles: (a) bare gold SPR chip surface, (b) patulin imprinted SPR nanosensor surface, and (c) non-imprinted SPR nanosensor surface.

The thicknesses of the patulin imprinted and the non-imprinted SPR nanosensor surfaces were obtained as $65.7 \mathrm{~nm}$ and $62.3 \mathrm{~nm}$, respectively. When the results of the characterization studies of the patulin imprinted and the non-imprinted SPR nanosensor surfaces were examined, it was proved that the synthesized nanoparticles spread homogeneously on the surface of SPR nanosensors.

\subsection{Kinetic studies}

Kinetic studies of the patulin imprinted and the non-imprinted SPR nanosensors were prepared in $\mathrm{pH} 6.0$ phosphate buffer solutions at concentrations of $0.5 \mathrm{nmol}$ - $750 \mathrm{nmol}$. In this study, kinetic analyses were performed using SPRimager II (GWC Technologies, Madison, WI, USA). The SPRimager system has a Kretschmann configuration and measures the angle of incident light at which SPR takes place. The real-time values of the SPR sensorgrams are given in Figs. 5(a) and 5(b). In the first step, the patulin imprinted SPR nanosensor was washed with $\mathrm{pH} 6.0$ phosphate buffer solution for $3 \mathrm{~min}$. Then, the prepared patulin solutions in the concentration range of $0.5 \mathrm{nmol}-750 \mathrm{nmol}$ were given to the SPRimager II system for $7 \mathrm{~min}$ and resonance frequency change $\Delta R(\%)$ values were determined for each kinetic analysis. Then, $0.5 \mathrm{~mol}$ $\mathrm{NaCI}$ solution was used to desorb the bound patulin molecules from the SPR nanosensor surface. The desorption step was performed at $3 \mathrm{~min}$ for each analysis. All kinetic data were calculated by using SPR view software.

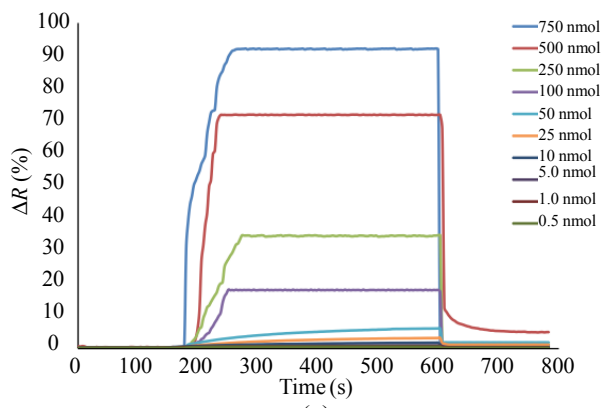

(a)

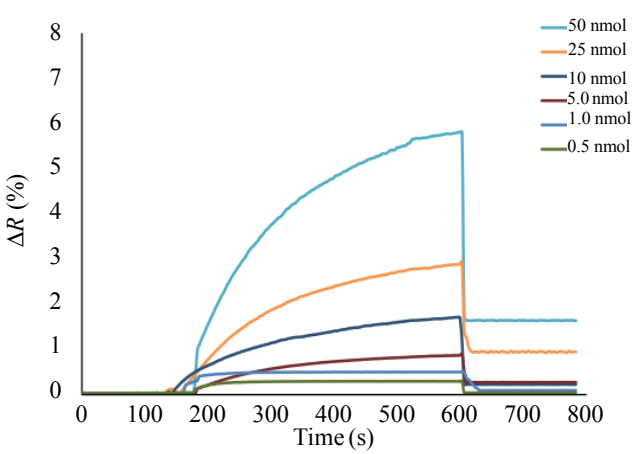

(b)

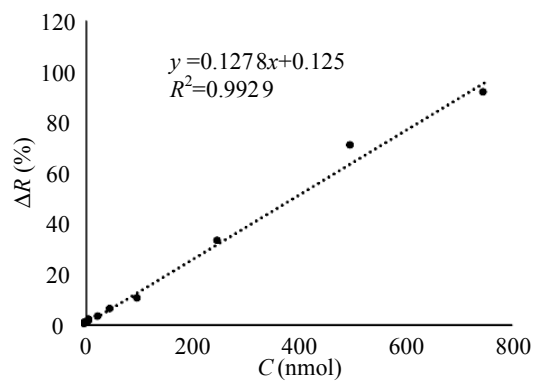

(c)

Fig. 5 Real-time responses [patulin concentration range of (a) between $0.5 \mathrm{nmol}$ and $750 \mathrm{nmol}$ and (b) between $0.5 \mathrm{nmol}$ and $50 \mathrm{nmol}$, and linear regions (c) of the patulin imprinted SPR nanosensor aqueous solutions of patulin at different concentrations].

As shown in Fig. 5(c), the resonance frequency 
change $[\Delta R(\%)]$ varied based on the plasmonic principle and the patulin concentration increased proportionally. The good linear equation between $0.5 \mathrm{nmol}$ and $750 \mathrm{nmol}$ concentration was $y=$ $0.1278 x+0.125$ with a determination coefficient of 0.992 . The limit of detection ( $\mathrm{LOD}=3.3 \mathrm{~S} / \mathrm{m}$ ) and quantification $(\mathrm{LOQ}=10 \mathrm{~S} / \mathrm{m})$ of patulin molecules were calculated based on the slope of the calibration curve and with $y=0.1278 x+0.125$ equation. $S$ and $m$ are the standard deviations of the intercept and slope of the regression line, respectively [34]. The LOD and LOQ for patulin detection were $0.011 \mathrm{nmol}$ and $0.036 \mathrm{nmol}$, respectively. Other sensor studies performed for patulin determination are given in Table 1 for comparison.

Table 1 Comparison of different sensor systems in the literature for patulin detection.

\begin{tabular}{|c|c|c|c|c|}
\hline Method & $\begin{array}{l}\text { Linear concentration range } \\
\text { (nmol) }\end{array}$ & Sample & $\begin{array}{l}\text { Limit of detection } \\
\text { (nmol) }\end{array}$ & Ref. \\
\hline Nanosensor & $430-6500$ & Apple juice & 320 & [43] \\
\hline Colorimetric aptasensor & $0.32-16$ & - & 0.31 & [44] \\
\hline Aptasensor & $0.0649-649$ & - & 0.0195 & {$[45]$} \\
\hline Surface plasmon resonance & $0-100$ & - & 0.1 & [46] \\
\hline Quartz crystal microbalance & - & Apple puree & 140 & {$[47]$} \\
\hline Surface-enhanced Raman spectroscopy & $0.5-1000$ & Blueberry and grapefruit jam, orange juice & $8.5 \times 10^{-2}$ & [48] \\
\hline Electrochemical sensor & $0.001-1.0$ & - & $7.57 \times 10^{-4}$ & {$[49]$} \\
\hline Electrochemical sensor & $0.012-12.99$ & Apple and grape juice & $6 \times 10^{-3}$ & {$[50]$} \\
\hline Electrochemical sensor & $0.1-10000$ & Apple juice & 0.03 & {$[51]$} \\
\hline Electrochemical aptasensor & $0.00032-3.25$ & Apple juice, wine & $2.6 \times 10^{-4}$ & {$[52]$} \\
\hline Electrochemical sensor & $50-600$ & Apple juice & 0.6635 & {$[53]$} \\
\hline Impedimetric Immunosensor & $0.065-65$ & Apple juice & 63.6 & {$[54]$} \\
\hline Electrochemical sensor & $0.0065-455$ & Apple juice & $4 \times 10^{-4}$ & {$[55]$} \\
\hline Surface plasmon resonance nanosensor & $0.5-750$ & Apple juice & 0.011 & This study \\
\hline
\end{tabular}

Mycotoxins formed during the processing of fruits into fruit juice can easily pass into fruit juice, since they have the ability to be dissolved in water. The most common mycotoxins in fruit juices and wines are PAT, OTA, and AFB1. In this study, OTA and AFB1 molecules were chosen as competitive agents to demonstrate the selectivity of the patulin imprinted and the non-imprinted SPR nanosensor. To compare the selectivity of the patulin imprinted SPR nanosensor, the non-imprinted SPR nanosensor was also prepared. For selectivity analysis, patulin concentrations were kept constant at $100 \mathrm{nmol}$. Figure 6 shows the responses of the SPR nanosensor for OTA and AFB1 molecules were lower than that of PAT. This is due to the selective cavities of patulin formed in patulin imprinted nanoparticles. The relative selectivity coefficients $\left(k^{\prime}\right)$ of the patulin imprinted SPR nanosensor for PAT/OTA and PAT/AFB1 were 9.753 times and 8.314 times,

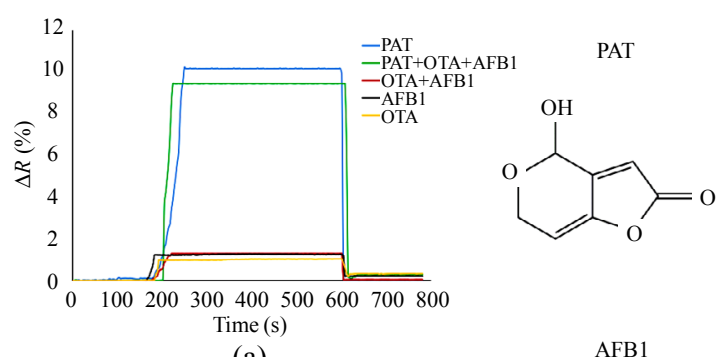

(a)

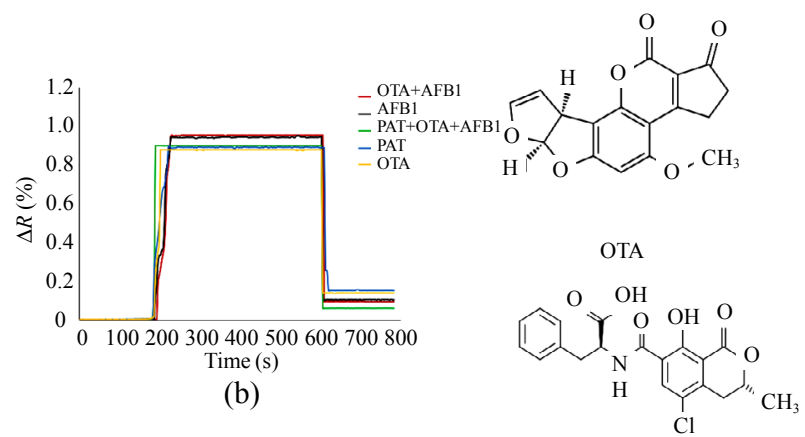

Fig. 6 Selectivity studies of the patulin imprinted (a) and the non-imprinted (b) SPR nanosensors, and chemical structures of PAT, OTA, and AFB1 molecules. 
respectively (Table 2). The relative selectivity coefficients $\left(k^{\prime}\right)$ results showed that the patulin imprinted SPR nanosensor had higher selectivity for PAT in comparison with OTA and AFB1. The resonance frequency change $\Delta R \quad(\%)$ of the non-imprinted SPR nanosensor with both PAT and other competitive agents as OTA and AFB1 were obtained as $0.892,0.879$, and 0.944 , respectively. When the selectivity results were examined, it showed that the patulin imprinted SPR nanosensors had higher selectivity than the non-imprinted SPR nanosensors.

Table 2 Selectivity and relative selectivity coefficients for PAT, OTA, and AFB1 molecules for the patulin imprinted and the non-imprinted SPR nanosensors.

\begin{tabular}{c|c|c|c|c|c}
\hline & \multicolumn{2}{|c|}{$\begin{array}{c}\text { MIP } \\
\text { nanosensor }\end{array}$} & \multicolumn{2}{c|}{$\begin{array}{c}\text { NIP } \\
\text { nanosensor }\end{array}$} & \\
\hline Molecules & $\Delta R$ & $k$ & $\Delta R$ & $k$ & $k^{\prime}$ \\
PAT & 10.128 & - & 0.892 & - & - \\
OTA & 1.023 & 9.900 & 0.879 & 1.015 & 9.753 \\
AFB1 & 1.289 & 7.857 & 0.944 & 0.945 & 8.314 \\
OTA+AFB1 & 1.338 & 7.569 & 0.957 & 0.932 & 8.121 \\
PAT+OTA+AFB1 & 9.420 & 1.075 & 0.899 & 0.992 & 1.084 \\
\hline
\end{tabular}

\subsection{Detection of patulin from apple juice}

After all kinetic analyses of the patulin imprinted SPR nanosensor, the amount of patulin in the apple juice was analyzed to show its applicability. The obtained extraction from apple juice was spiked from the patulin aqueous solution at concentrations of $50 \mathrm{nmol}$ and $100 \mathrm{nmol}$. In the kinetic studies, the patulin imprinted SPR nanosensor was first equilibrated with the phosphate buffer $(\mathrm{pH} \mathrm{6.0)}$ for $3 \mathrm{~min}$. The spiked patulin apple juice samples were applied to the SPR system for $7 \mathrm{~min}$. The removal of patulin molecule from the patulin imprinted SPR nanosensor surface was carried out with $0.5 \mathrm{~mol}$ $\mathrm{NaCI}$ solution for $3 \mathrm{~min}$ (Fig. 7). The obtained kinetic analysis results from the SPR system with the obtained results from LC-MS measurements are compared in Table 3 . Table 3 shows the recovery (\%) for determining the reliability and accuracy of both the patulin imprinted SPR nanosensor and LC-MS analysis results. Considering the results, the consistency of SPR nanosensor results and LC-MS analysis results with each other shows that the patulin imprinted SPR nanosensor is quantitative, accurate, reliable, and sensitive for patulin detection in apple juice.

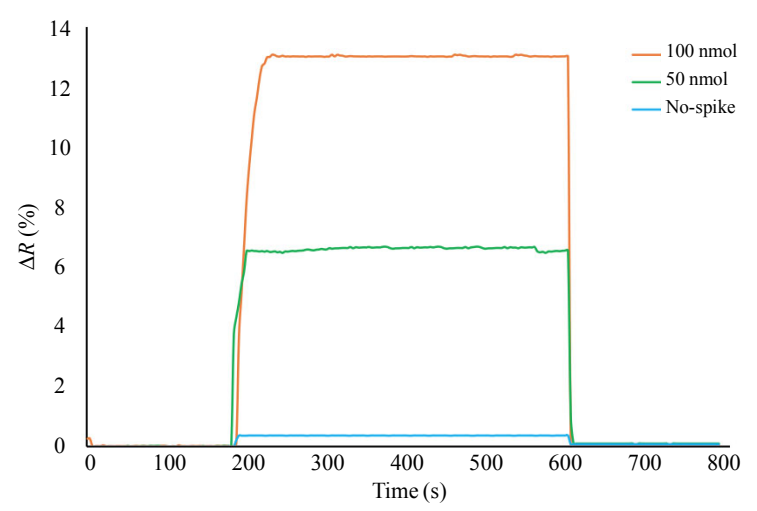

Fig. 7 Kinetic analysis for patulin detection in apple juice samples.

Table 3 Recoveries of patulin in apple juice.

\begin{tabular}{c|c|c|c|c}
\hline \multirow{2}{*}{$\begin{array}{c}\text { Added TA } \\
(\mathrm{nmol})\end{array}$} & \multicolumn{2}{|c|}{$\begin{array}{c}\text { Found PAT } \\
(\mathrm{nmol})\end{array}$} & \multicolumn{2}{c}{$\begin{array}{c}\text { Recovery } \\
(\%)\end{array}$} \\
\cline { 2 - 5 } & $\begin{array}{c}\text { SPR } \\
\text { nanosensor }\end{array}$ & LC-MS & $\begin{array}{c}\text { SPR } \\
\text { nanosensor }\end{array}$ & LC-MS \\
\hline 50 & $49.13 \pm 0.011$ & $49.01 \pm 0.056$ & $98.24 \pm 0.021$ & $98.03 \pm 0.113$ \\
100 & $98.32 \pm 0.121$ & $98.27 \pm 0.369$ & $98.32 \pm 0.124$ & $98.26 \pm 0.369$ \\
\hline
\end{tabular}

\subsection{Reusability}

One of the major advantages of molecularly imprinted SPR nanosensors is that they can be reused under long-term storage conditions without any performance loss. As shown in Fig. 8(a), the reusability of the patulin imprinted SPR nanosensor was tested by using the same patulin concentration solution (50 nmol) and same SPR chip in five equilibration, adsorption, and desorption cycles. Reusability of the patulin imprinted SPR nanosensor was also tested in different times. The patulin imprinted SPR sensors were kept at $4{ }^{\circ} \mathrm{C}$ and there was no significant instability in the SPR responses against patulin after 6 months keeping. After 6 months, the storage stability and efficiency of the patulin imprinted SPR nanosensor were tested in the presence of $50 \mathrm{nmol}$ patulin solution [Fig. 8(b)]. It was observed that the initial activity decreased by $12.20 \%$. 


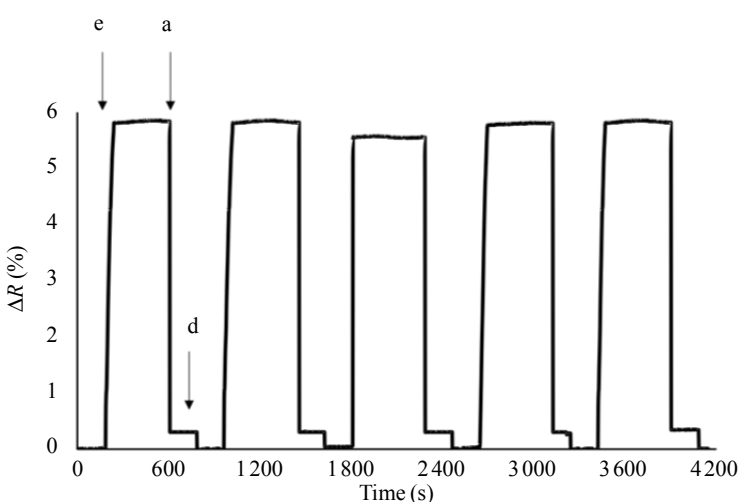

(a)

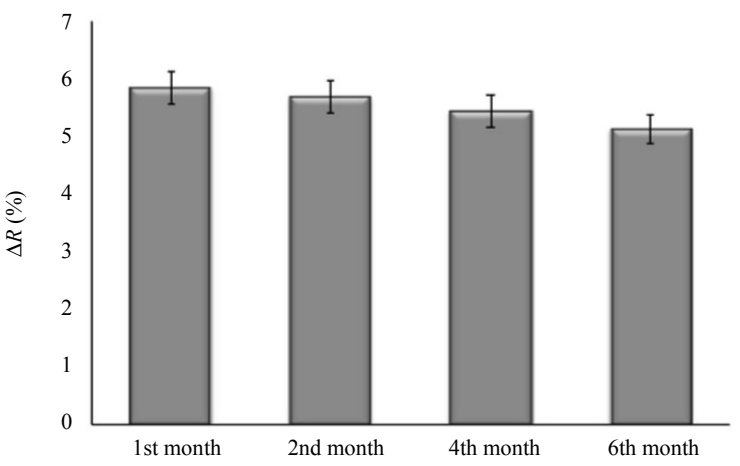

(b)

Fig. 8 Reusability (a) and short-term and long-term stabilities (b) of the patulin imprinted SPR nanosensors (e: equilibration; a: adsorption; d:desorption cycles).

\section{Conclusions}

Mycotoxin contamination in foods is a world-wide serious problem, and chronic exposure at low doses causes several health issues. Patulin, which is from the mycotoxins group, is an important metabolite in the structure of polychitide produced by the number of molds. The permitted maximum limits in various foods and their monitoring and control are necessary to prevent poisoning of humans and animals. Therefore, sensitive methods are required to achieve very low detection limits for the contamination at low concentrations. In this study, a cheap, simple, rapid, reusable, and sensitive patulin imprinted SPR nanosensor was designed for the detection of patulin in apple juice. The limits of detection and quantification values of the patulin imprinted SPR nanosensor were determined as $0.011 \mathrm{nmol}$ and $0.036 \mathrm{nmol}$, respectively. Selectivity of the patulin imprinted SPR nanosensor was tested by using two different competing molecules such as OTA and AFB1. Selectivity results showed that the cavities formed in the patulin imprinted nanoparticles were sensitive to PAT rather than those to OTA and AFB1 at coefficients, such as 9.753 and 8.314 , respectively. The reusability of the patulin imprinted SPR nanosensors was determined as $87.80 \%$. In addition, the patulin imprinted SPR nanosensor has an informal usage more economical with its easy and reusable features and its storage capacity is longer than those of other detection methods.

\section{Acknowledgment}

This study was partly supported by Scientific Research Foundation of Hacettepe University Project (Grant No. FHD-2019-18247).

Open Access This article is distributed under the terms of the Creative Commons Attribution 4.0 International License (http://creativecommons.org/licenses/by/4.0/), which permits unrestricted use, distribution, and reproduction in any medium, provided you give appropriate credit to the original author(s) and the source, provide a link to the Creative Commons license, and indicate if changes were made.

\section{References}

[1] K. De Ruyck, M. De Boevre, I. Huybrechts, and S. De Saeger, "Dietary mycotoxins, co-exposure, and carcinogenesis in humans: short review," Mutation Research, 2015, 766: 32-41.

[2] M. Champdore, P. Bazzicalupo, L. Napoli, D. Montesarchio, G. Fabio, I. Cocozza, et al., "A new competitive fluorescence assay for the detection of patulin toxin," Analytical Chemistry, 2007, 79(2): 751-757.

[3] P. Regal, M. Díaz-Bao, R. Barreiro, C. Fente, and A. Cepeda, "Design of a molecularly imprinted stir-bar for isolation of patulin in apple and LC-MS/MS detection," Separations, 2017, 4(2): 11.

[4] X. Song, D. Wang, and M. Kim, "Development of an immuno-electrochemical glass carbon electrode sensor based on graphene oxide/gold nanocomposite and antibody for the detection of patulin," Food Chemistry, 2021, 342: 128257.

[5] X. Li, H. Li, X. Li, and Q. Zhang, "Determination of trace patulin in apple-based food matrices," Food Chemistry, 2017, 233: 290-301. 
[6] O. P. Omotayo, A. O. Omotayo, M. Mwanza, and O. O. Babalola, "Prevalence of mycotoxins and their consequences on human health," Toxicology Research, 2019, 35(1): 1-7.

[7] S. Ramalingam, A. Bahuguna, and M. Kim, "The effect of mycotoxin patulin on cells and cellular components," Trends in Food Science and Technology, 2019, 83: 99-113.

[8] I. Sadok, A. Szmagara, and M. M. Staniszewska, "The validated and sensitive HPLC-DAD method for determination of patulin in strawberries," Food Chemistry, 2018, 245: 364-370.

[9] Y. Yu and Z. Fan, "Determination of patulin in apple juice using magnetic solid-phase extraction coupled with high-performance liquid chromatography," Food Additives Contaminants: Part A, 2017, 34(2): 273-281.

[10] X. Li, H. Li, W. Ma, Z. Guo, X. Li, X. Li, et al., "Determination of patulin in apple juice by single-drop liquid-liquid-liquid microextraction coupled with liquid chromatography-mass spectrometry," Food Chemistry, 2018, 257: 1-6.

[11] "Commission Regulation, No.1881/2006 of 19 December 2006 setting maximum levels for certain contaminants in foodstuffs," Official Journal of the European Union, 2006, L364, pp.5.

[12] J. Sun, W. Guo, J. Ji, Z. Li, X. Yuan, F. Pi, et al., "Removal of patulin in apple juice based on novel magnetic molecularly imprinted adsorbent $\mathrm{Fe}_{3} \mathrm{O}_{4} @ \mathrm{SiO}_{2} @ \mathrm{CS}-\mathrm{GO} @ \mathrm{MIP},{ }^{\prime}$ Food Science and Technology, 2020, 118: 108854.

[13] C. Wei, Q. Zhai, L. Yu, N. Qiao, J. Zhao, H. Zhang, et al., "Progress in the distribution, toxicity, control, and detoxification of patulin: a review," Toxicon, 2020, 184: 83-93.

[14] A. Vidal, S. Ouhibi, R. Ghali, A. Hedhili, S. D. Saeger, and M. D. Boevre, "The mycotoxin patulin: An updated short review on occurrence, toxicity and analytical challenges," Food and Chemical Toxicology, 2019, 129: 249-256.

[15] R. Funari, B. D. Venture, R. Carrieri, L. Morra, E. Lahoz, F. Gesuele, et al., "Detection of parathion and patulin by quartz-crystal microbalance functionalized by the photonics immobilization technique," Biosensors and Bioelectronics, 2015, 67: 224-229.

[16] B. He and X. Lu, "An electrochemical aptasensor based on tetrahedral DNA nanostructures as a signal probe carrier platform for sensitive detection of patulin," Analytica Chimica Acta, 2020, 1138: 123-131.

[17] S. Ouhibi, A. Vidal, C. Martins, R. Gali, A. Hedhili, S. De Saeger, et al., "LC-MS/MS methodology for simultaneous determination of patulin and citrinin in urine and plasma applied to a pilot study in colorectal cancer patients," Food and Chemical Toxicology, 2020, 136: 110994.
[18] D. Moreno-González, P. Jáč, P. Riasová, and L. Nováková, "In-line molecularly imprinted polymer solid phase extraction-capillar electrophoresis coupled with tandem mass spectrometry for the determination of patulin in apple-based food," Food Chemistry, 2021, 334: 127607.

[19] I. Sadok, A. Stachniuk, and M. Staniszewska, "Developments in the monitoring of patulin in fruits using liquid chromatography: an overview," Food Analytical Methods, 2019, 12: 76-93.

[20] N. Kharandi, M. Babri, and J. Azad, "A novel method for determination of patulin in apple juices by GC-MS," Food Chemistry, 2013, 141(3): 1619-1623.

[21] Y. Saylan, S. Akgönüllü, H. Yavuz, S. Ünal, and A. Denizli, "Molecularly imprinted polymer based sensors for medical applications," Sensors, 2019, 19(6): 1279

[22] J. Chen, C. Yang, P. Gu, Y. Kuang, C. Tang, S. Chen, et al., "High sensing properties of magnetic plasmon resonance by strong coupling in three-dimensional metamaterials," Journal of Lightwave Technology, 2021, 39(2): 562-565.

[23] J. Chen, Y. Kuang, P. Gu, S. Feng, Y. Zhu, C. Tang, et al., "Strong magnetic plasmon resonance in a simple metasurface for high-quality sensing," Journal of Lightwave Technology, 2021, 39(13): 4525-4528.

[24] J. Chen, S. Chen, P. Gu, Z. Yan, C. Tang, Z. Xu, et al., "Electrically modulating and switching infrared absorption of monolayer graphene in metamaterials," Carbon, 2020, 162: 187-194.

[25] J. Chen, C. Peng, S. Qi, Q. Zhang, C. Tang, X. Shen, et al., "Photonic microcavity-enhanced magnetic plasmon resonance of metamaterials for sensing applications," IEEE Photonics Technology Letters, 2019, 31(2): 113-116.

[26] J. Chen, S. Qi, X. Hong, P. Gu, R. Wei, C. Tang, et al., "Highly sensitive 3D metamaterial sensor based on diffraction coupling of magnetic plasmon resonances," Results in Physics, 2019, 15: 102791.

[27] J. Chen, H. Nie, C. Tang, Y. Cui, B. Yan, Z. Zhang, et al., "Highly sensitive refractive-index sensor based on strong magnetic resonance in metamaterials," Applied Physics Express, 2019, 12(5): 052015.

[28] J. Chen, H. Nie, C. Peng, S. Qi, C. Tang, Y. Zhang, et al., "Enhancing the magnetic plasmon resonance of three-dimensional optical metamaterials via strong coupling for high-sensitivity sensing," Journal of Lightwave Technology, 2018, 36(16): 3481-3485.

[29] A. S. Kushwaha, A. Kumar, R. Kumar, and S. K. Srivastava, "A study of surface plasmon resonance (SPR) based biosensor with improved sensitivity," Photonics and Nanostructure-Fundamentals and Applications, 2018, 31: 99-106. 
[30] S. Zeng, D. Baillargeat, H. P. Ho, and K. T. Yong, "Nanomaterials enhanced surface plasmon resonance for biological and chemical sensing applications," Chemical Society Reviews, 2014, 43(10): 3426-3452.

[31] E. Helmerhorst, D. J. Chandler, M. Nussio, and C. D. Mamotte, "Real-time and label-free bio-sensing of molecular interactions by surface plasmon resonance: a laboratory medicine perspective," Clinical Biochemist Reviews, 2012, 33(4): 161-173.

[32] D. Çimen and A. Denizli, "Development of rapid, sensitive, and effective plasmonic nanosensor for the detection of vitamins in infact formula and milk samples," Photonic Sensors, 2020, 10(4): 316-332.

[33] A. Rahtuvanoğlu, S. Akgönüllü, S. Karacan, and A. Denizli, "Biomimetic nanoparticles based surface plasmon resonance biosensors for histamine detection in foods," ChemistrySelect, 2020, 5(19): 5683-5692.

[34] N. Bereli, D. Çimen, S. Hüseynli, and A. Denizli, "Detection of amoxicillin residues in egg extract with a molecularly imprinted polymer on gold microchip using surface plasmon resonance and quartz crystal microbalance methods," Journal of Food Science, 2020, 85(12): 4152-4160.

[35] S. Akgönüllü, H. Yavuz, and A. Denizli, "SPR nanosensor based on molecularly imprinted polymer film with gold nanoparticles for sensitive detection of aflatoxin B1," Talanta, 2020, 219, (1): 121219.

[36] D. Çimen, N. Bereli, H. Yavuz, and A. Denizli, "Chapter 8: Sensors for the detection of food," in Nanosensors for Environment, Food and Agriculture Vol. 1. Editors: Vineet Kumar, Praveen Guleria Shivendu Ranjan, Nndita Dasgupta, and Eric Lichtfouse. Switzerland AG: Springer Nature, 2021: 169-182.

[37] D. Çimen, N. Bereli, M. Andaç, and A. Denizli, "Molecularly imprinted cryogel columns for Concanavalin A purification from jack bean extract," Separation Science Plus, 2018, 1(6): 454-463.

[38] L. Chen, X. Wang, W. Lu, X. Wu, and J. Li, "Molecular imprinting: perspectives and applications," Chemical Society Reviews, 2016, 45(8): 2137-2211.

[39] A. Rico-Yuste and S. Carrasco, "Molecularly imprinted polymer-based hybrid materials for the development of optical sensors," Polymer, 2019, 11(7): 1173.

[40] S. Faalnouri, D. Çimen, N. Bereli, and A. Denizli, "Surface plasmon resonance nanosensors for detecting amoxicillin in milk samples with amoxicillin imprinted poly (hydroxyethyl methacrylateN-methacryloyl-(L)-glutamicacid)," ChemistrySelect, 2020, 5(15): 4761-4769.

[41] W. Zhang, Y. Han, X. Chen, X. Luo, J. Wang, T. Yue, et al., "Surface molecularly imprinted polymer capped Mn-doped $\mathrm{ZnS}$ quantum dots as a phosphorescent nanosensor for detecting patulin in apple juice," Food Chemistry, 2017, 232: 145-154.

[42] M. Zhao, H. Shao, Y. He, H. Li, M. Yan, Z. Jiang, et al., "The determination of patulin from food samples using dual-dummy molecularly imprinted solid-phase extraction coupled with LC-MS/MS," Journal of Chromatography B, 2019, 1125: 121714.

[43] Y. Zhou, C. Gao, and C. Y. Guo, "UV assisted ultrasensitive trace $\mathrm{NO}_{2}$ gas sensing based on few-layer $\mathrm{MoS}_{2}$ nanosheet-ZnO nanowire heterojunctions at room temperature," Journal of Materials Chemistry A, 2018, 6(22): 10286-10296.

[44] S. Wu, N. Duan, W. Zhang, S. Zhao, and Z. Wang, "Screening and development of DNA aptamers as capture probes for colorimetric detection of patulin," Analytical Biochemistry, 2016, 508: 58-64.

[45] Z. Wu, E. Xu, Z. Jin, and J. Irudayaraj, “An ultrasensitive aptasensor based on fluorescent resonant energy transfer and exonuclease-assisted target recycling for patulin detection," Food Chemistry, 2018, 249: 136-142.

[46] A. Pennacchio, G. Ruggiero, M. Staiano, G. Piccialli, G. Oliviero, A. Lewkowicz, et al., "A surface plasmon resonance based biochip for the detection of patulin toxin," Optical Materials, 2014, 36(10): 1670-1675.

[47] R. Funari, B. D. Ventura, R. Carrieri, L. Morra, E. Lahoz, F. Gesuele, et al., "Detection of parathion and patulin by quartz-crystal microbalance functionalized by the photonics immobilization technique," Biosensors and Bioelectronics, 2015, 67: 224-229.

[48] Y. Zhu, L. Wu, H. Yan, Z. Lu, W. Yin, and H. Han, "Enzyme induced molecularly imprinted polymer on SERS substrate for ultrasensitive detection of patulin," Analytical Chimica Acta, 2019, 1101: 111-119.

[49] W. Guo, F. Pi, H. Zhang, J. Sun, Y. Zhang, and $\mathrm{X}$. Sun, "A novel molecularly imprinted electrochemical sensor modified with carbon dots, chitosan, gold nanoparticles for the determination of patulin," Biosensors and Bioelectronics, 2017, 98: 299-304.

[50] Q. Huang, Z. Zhao, D. Nie, K. Jiang, W. Guo, K. Fan, et al., "Molecularly imprinted poly(thionine)-based electrochemical sensing platform for fast and selective ultratrace determination of patulin," Analytical Chemistry, 2019, 91(6): 4116-4123.

[51] J. Xu, X. Qiao, Y. Wang, Q. Sheng, T. Yue, J. Zheng, et al., "Electrostatic assembly of gold nanoparticles on black phosphorus nanosheets for electrochemical aptasensing of patulin," Microchimical Acta, 2019, 186(4): 238.

[52] B. He and X. Dong, "Nb.BbvCI powered DNA 
walking machine-based Zr-MOFs-labeled electrochemical aptasensor using Pt@AuNRs/ Fe-MOFs/PEI-rGO as electrode modification material for patulin detection," Chemical Engineering Journal, 2021, 405: 126642.

[53] S. Shukla, Y. Haldorai, I. Khan, S. Kang, C. H. Kwak, S. Gandhi, et al., "Bioreceptor-free, sensitive and rapid electrochemical detection of patulin fungal toxin, using a reduced graphene oxide@ $\mathrm{SnO}_{2}$ nanocomposite," Materials Science Engineering $C$, 2020, 113: 110916.
[54] W. I. Riberi, M. A. Zon, H. Fernández, and F. J. Arévalo, "Impedimetric immunosensor to determine patulin in apple juices using a glassy carbon electrode modified with graphene oxide," Microchemical Journal, 2020, 158: 105192.

[55] B. Hatamluyi, M. Rezayi, H. R. Beheshti, and M. T. Boroushaki, "Ultra-sensitive molecularly imprinted electrochemical sensor for patulin detection based on a novel assembling strategy using Au@Cu-MOF/NGQDs," Sensors Actuators: B. Chemical, 2020, 318 : 128219. 